\title{
A Word about Sources
}

This collection draws on a wide range of source material - much of which is freely available over the internet. Rather than list this material and provide what I hope will be a static web links, I decided to let search engines help me keep the sources up to date. Wherever I reference something, I try to provide enough information for an interested reader to find it. For anyone determined to track down all my original source material, I would be happy to provide an unedited early draft of this collection with all the notes and web references in place. 